\title{
Synthesis and characterization of a paramagnetic [2]rotaxane based on a crown ether-like wheel incorporating a nitroxide motif
}

\author{
Valentina Bleve, ${ }^{[a]}$ Paola Franchi, ${ }^{[a]}$ Evangelia Konstanteli, ${ }^{[a][b]}$ Lorenzo Gualandi, ${ }^{[a]}$ Stephen M. \\ Goldup, ${ }^{[c]}$ Elisabetta Mezzina, ${ }^{*[a]}$ and Marco Lucarini ${ }^{*[a]}$
}

\begin{abstract}
The synthesis of a new nitroxide crown ether (8) and its use as the wheel in a bistable [2] rotaxane containing dialkylammonium and 4,4-bipyridinium recognition sites, is reported. The synthesis of $\mathbf{8}$ was achieved by the sequential addition of substituted phenyl groups to nitrone derivatives leading to the preferential formation of the cis-stereoisomer. Due to charge-dipolar interactions between the nitroxide unit and bipyridinium moiety, it was possible to probe the movement of the macrocycle between the two molecular stations of the [2] rotaxane after addition of a base by measuring the nitrogen hyperfine splittings in the corresponding EPR spectra. The equilibrium constant for the complexation of dibenzyl viologen by macrocycle $\mathbf{8}$ was also determined by EPR titration.
\end{abstract}

Nitroxide radicals are an important group of open-shell molecules with intriguing catalytic and magnetic properties, giving rise to diverse and important applications in many fields of chemistry and related sciences. These applications include the biomedical field (diagnostic spin probes, ${ }^{[1]}$ spin traps, ${ }^{[2]}$ and potential antioxidant therapeutics), ${ }^{[3]}$ the development of sustainable chemical processes (catalysts in aerobic oxidation ${ }^{[4]}$ and material chemistry (radical initiators for living and other controlled polymerizations). ${ }^{[5]}$

However, with the notable exception of the alkyl-nitronyl nitroxide organic magnet systems, ${ }^{[6]}$ the use of nitroxide in molecular-level assembly processes remains largely unexplored, certainly in comparison with the use of ethers, amides, imines, ureas, and guanidinium units. ${ }^{[7]}$ This is somewhat surprising given their desirable intrinsic properties: nitroxides possess one of the largest dipole moments known for any functional-group (close to $3 \mathrm{D}$ ), ${ }^{[8]}$ making them potentially useful for control of molecular orientation: the $\mathrm{N}-\mathrm{O} \bullet$ motif is a good hydrogen ${ }^{[9]}$ and halogen-bond ${ }^{[10]}$ acceptor and they offer a simple reversible route to diamagnetic species via one-electron electrochemical oxidation ${ }^{[11]}$ Rotaxanes and catenanes containing nitroxide units have been described in the literature, ${ }^{[12]}$ but in all cases the paramagnetic unit was used as spin probe and linked to the macrocycle or to the thread without participating to the recognition process. In most of these examples spin exchange

[a] Dr. V. Bleve, Dr. P. Franchi E. Konstanteli, L. Gualandi, Prof. Dr. E. Mezzina, Prof. M. Lucarini

Department of Chemistry "Giacomo. Ciamician"

University of Bologna, Via S. Giacomo 11, 40126 Bologna (Italy)

E-mail: marco.lucarini@unibo.it

[b] E. Konstanteli

Nationaland Kapodistrian University of Athens, (Greece)

[c] Prof. Dr. S.Goldup

Department of Chemistry, University of Southampton

Southampton, SO17 1BJ (UK)

Supporting information for this article is given via a link at the end of the document interaction between two radical units was used to probe the shuttling process of the macrocycle between two different recognition sites triggered by protonation or electron transfer. ${ }^{[12]}$ While the dimerization of radical ions has been proposed to move a macrocyclic ring between different molecular recognition sites in a [2]rotaxane, ${ }^{[13]}$ to the best of our knowledge no examples of mechanical interlocked molecules like rotaxanes or catenanes in which a nitroxide functionality could be used as recognition unit has been reported so far.

Based on this, we decided to prepare and characterize the first example of a nitroxide-containing rotaxane in which the $\mathrm{N}-\mathrm{O} \bullet$ motif acts not only as EPR probe for the shuttling process, but could be also directly involved in the recognition process of a molecular site. This was achieved by inserting a dialkyl nitroxide functionality in a crown ether-like frame containing seven ethereal oxygen atoms (8, Scheme 1$)$ and by using this macrocycle for complexation of cationic guests. We will show that the EPR read-out discriminates between dialkylammonium $\left(\mathrm{NH}_{2}{ }^{+}\right)$and 4,4'-bipyridinium (BPY ${ }^{2+}$ ) recognition sites and this favourable feature can be used to probe shuttling process of the paramagnetic wheel in a bistable [2]rotaxane.

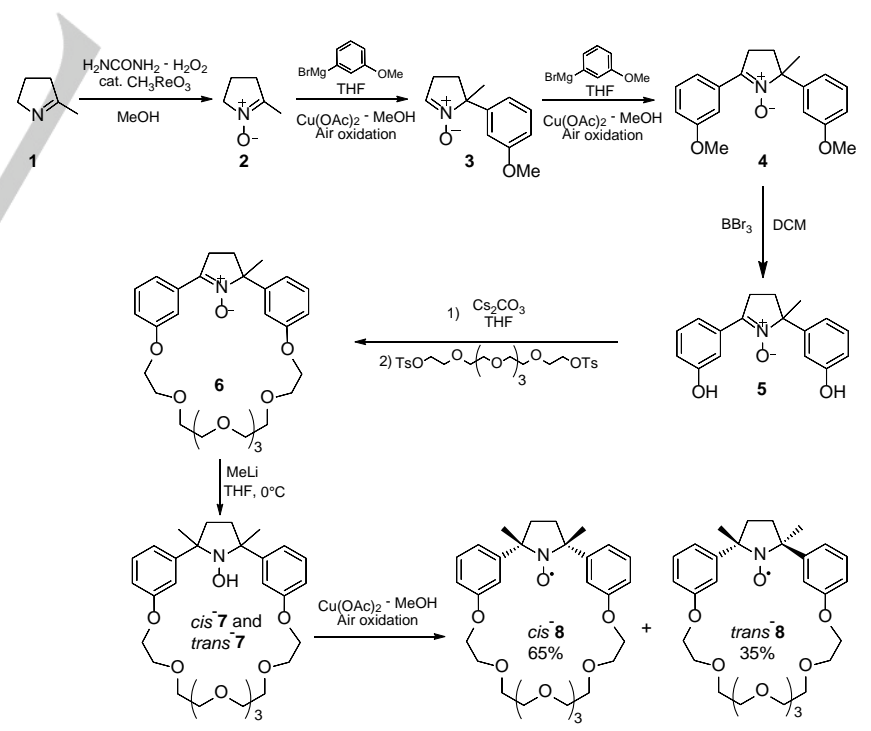

Scheme 1. Reaction scheme for the preparation of the paramagnetic macrocycle 8 .

Nitroxide based crown ether-like macrocycles are not new and were reported as far back as 1983 by Keana and coworkers. ${ }^{[14]}$ In such examples, the crown ether is inserted in both the aromatic rings at the ortho position in a 2,5-dimethyl-2,5diphenylpyrrolidine- $\mathrm{N}$-oxyl radical. In order to reduce the steric hindrance around the nitroxidic functionality, we decided to 
prepare a new derivative (8) where the two aromatic rings are linked via a meta-substitution to the nitroxide and to the polyether moieties. By adapting the original procedure proposed by Keana et al. ${ }^{[14]}$ for the preparation of the ortho-derivative, we prepared the new meta-substituted macrocycle $\mathbf{8}$ as described in Scheme 1. An important point which deserves a comment concerns the reaction of nitrone 6 with MeLi that provided hydroxylamine derivative 7 in quantitative yield as a mixture of cis and trans isomers in 6.5:3.5 molar ratio, as assessed by ${ }^{1} \mathrm{H}$ NMR (see Supporting Information). Although we were not able to obtain a single crystal of $\mathbf{7}$ for X-ray structure determination, we attributed the stereochemistry of the main isomer to the cis form by following the model presented by Shibata et al. reporting that an organometallic reagent attacks the pyrrolidine nitrone from the less hindered side. ${ }^{[15]}$ In our case, MeLi is expected to add to nitrone $\mathbf{6}$ from the side opposite to the bulky phenyl group to give the cis-hydroxylamine 7 .

The mixture of the two isomers (cis- and trans-7) was oxidized with air in the presence of $\mathrm{Cu}^{2+}$ ions. After purification of the crude mixture by column chromatography, a main fraction containing the nitroxide isomer cis-8 was recovered together with less abundant fractions corresponding to the other isomer of nitroxide (trans-8) and unreacted trans-7. Assignment of the cis-stereochemistry to the main fraction was obtained by NMR analysis after its reduction with phenylhydrazine. ${ }^{[16]}$ Interestingly, the two nitroxide isomers show different relative rates of reduction. While cis-8 isomer can be reduced to the corresponding hydroxylamine in 10-15 minutes, the trans-isomer is much more resistant toward reduction, requiring several days. This observation is in agreement with a previous report on transazethoxyl acid which was found to be the most resistant toward reduction if compared to analogous cis-derivatives. ${ }^{[17]}$ These findings corroborate the hypothesis previously exposed on the attribution of the stereoisomers. On this basis, the presence of unreacted trans-7 after oxidation of the mixture of hydroxylamines 7 suggests that also this step occurs more efficiently for the cis than the trans isomer because of the less steric hindrance around the $\mathrm{N}-\mathrm{OH}$ functionality in the former one. The $\mathrm{CH}_{2} \mathrm{Cl}_{2}$ solution of macrocycle cis-8 shows a typical nitroxide three line EPR spectrum and is characterized by $a_{N}=$ $14.35 \mathrm{G}$ and $g=2.0061$. EPR analysis of the $\mathrm{CH}_{2} \mathrm{Cl}_{2}$ solution containing trans-8 was virtually identical, apart a very small difference in the nitrogen coupling $\left(a_{N}=14.39 \mathrm{G}\right.$, see Supporting Information).

The host properties of the most abundant isomer, cis-8, towards cations which are known to be selectively complexed by crown ether derivatives, were then investigated by EPR. $\mathrm{Na}^{+}$and $\mathrm{K}^{+}$picrate salts are insoluble in $\mathrm{CH}_{2} \mathrm{Cl}_{2}$. However when a solution containing an excess of cis-8 was added to the suspension complete solubilisation of metal salts was observed. This clearly suggests that the paramagnetic macrocycle cis-8 maintains the complexation ability of crown ethers towards alkaline cations. Unexpectedly, the EPR spectra of the nitroxide cis-8 recorded in the presence of alkaline metal cations did not show any significant variation in the spectroscopic parameters compared to those measured in the absence of charged guests. The striking conclusion is that despite the position of the nitroxide group with respect to the ion binding cavity, the radical unit is not participating to the complexation process of the metal cation (which thus is expected to interact only with crown ether moiety, maintaining the cation far from the nitroxide). A similar result was obtained in the presence of dibenzyl ammonium $\mathrm{PF}_{6}$ salt which did not produce a significant change in the EPR spectrum of cis-8. Stochastic dynamic (SD) simulations performed in vacuo at $298 \mathrm{~K}$ on the dibenzylammonium@cis-8 complexes by using the AMBER* force field (see Supporting Information), suggested that the radical unit is indeed not participating to the complexation process of the ammonium group (see Figure 1a).

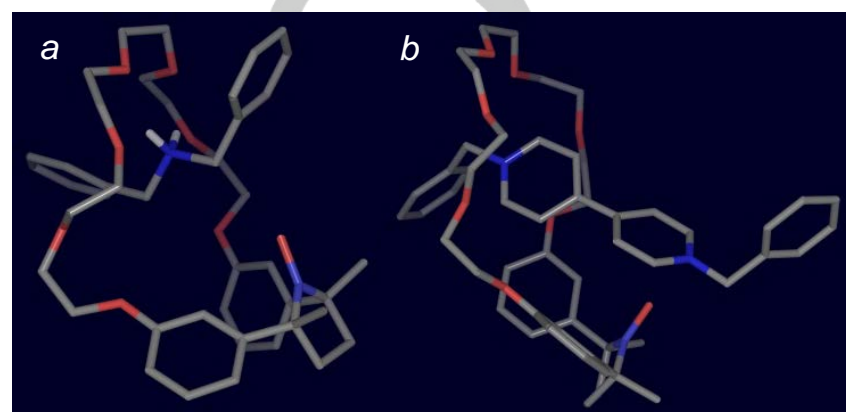

Figure 1. Stochastic dynamic simulations snapshots providing the possible structures of dibenzylammonium@cis-8 (a) and DBV@cis-8 (b) complexes.

A different spectroscopic behaviour was instead observed when the EPR spectrum of cis-8 was recorded in the presence of bis-cation dibenzyl viologen (DBV) as the $\mathrm{PF}_{6}$ salt. In this case the complete solubilization of the bis-pyridinium salt was accompanied by an increase of the nitrogen coupling $\left(a_{N}\right)$ value in the EPR spectrum of cis-8. As an example, in acetone at room temperature $a_{N}$ increases from $14.17 \mathrm{G}$ to $14.32 \mathrm{G}$ in the presence of $0.042 \mathrm{M} \mathrm{DBV}$.

Although the nature of the interaction is not fully defined, SD calculations (Figure $1 \mathrm{~b}$ ) suggests that binding of the bispyridinium salt occurs such that in the complex, one of the two cationic portions is interacting with the nitroxide unit by a chargedipolar interaction. In this model, the increase of $a_{N}$ can be explained on the basis of the two canonical forms I and II of the nitroxide function (Scheme 2). The greater the interaction with the positive charge of pyridinium unit, the more favoured will be structure II, bearing larger electron density on the oxygen and spin density on the nitrogen atom.

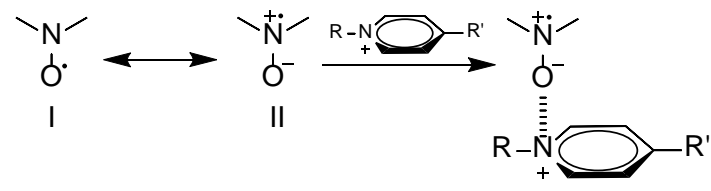

Scheme 2. Charge-dipole interaction between a dialkyl nitroxide and pyridinium unit.

As shown in Figure 2 the changes in the value of $a_{N}$ as a function of DBV concentration were well-modelled in acetone at $298 \mathrm{~K}$ by a $1: 1$ binding model using standard curve fitting methods and $K_{\text {eq, }}$ was found to be $69 \pm 19 \mathrm{M}^{-1} \cdot{ }^{[18]}$ This value is 
very close to that $\left(K_{\mathrm{eq}}=82 \mathrm{M}^{-1}\right)$ measured in $\mathrm{ACN}$ at $298 \mathrm{~K}$ for the complexation of DBV by the closely related diamagnetic macrocycle dibenzo[24]crown-8. ${ }^{[19]}$

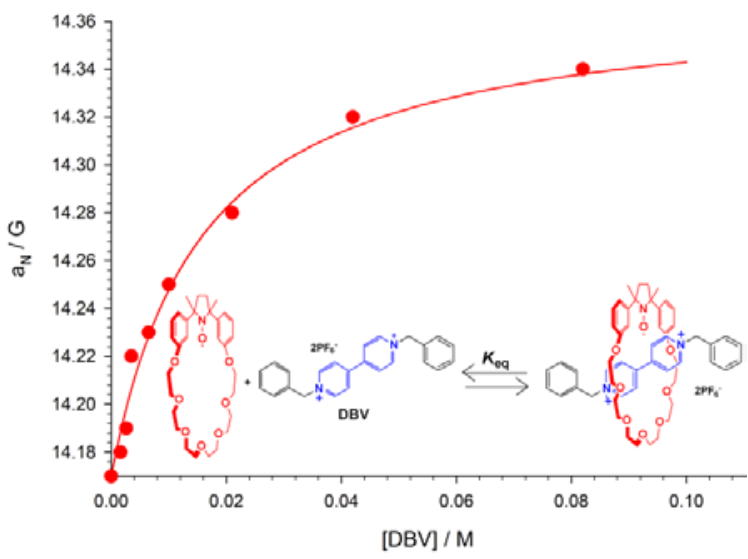

Figure 2. Plot of the EPR nitrogen coupling, $a_{\mathrm{N}}$, in cis-8 $(0.05 \mathrm{mM})$ versus the concentration of DBV at $298 \mathrm{~K}$ in acetone. The line represents the theoretical dependence of $a_{N}$ on DBV concentration calculated by taking into account the formation of $1: 1$ complex and $K_{\text {eq }}=69 \mathrm{M}^{-1}$.

Because the EPR read-out discriminates between different cationic guests we evaluated the possibility of using radical macrocycle cis-8 in a bistable [2]rotaxane containing dialkylammonium and 4,4-bipyridinium recognition sites in the thread. The rotaxane $\mathbf{1 1 H}_{\mathbf{1}} \mathbf{3} \mathbf{P F}_{6}$ (see Scheme 3) was prepared by following the classical threading-stoppering approach, which involves the in situ self-assembly of a pseudorotaxane, $10 \mathrm{H}_{2} \mathrm{PF}_{6}$, between the ring cis-8 and the half-dumbbell intermediate $\mathbf{9 H} \bullet 2 \mathrm{PF}_{6}$.

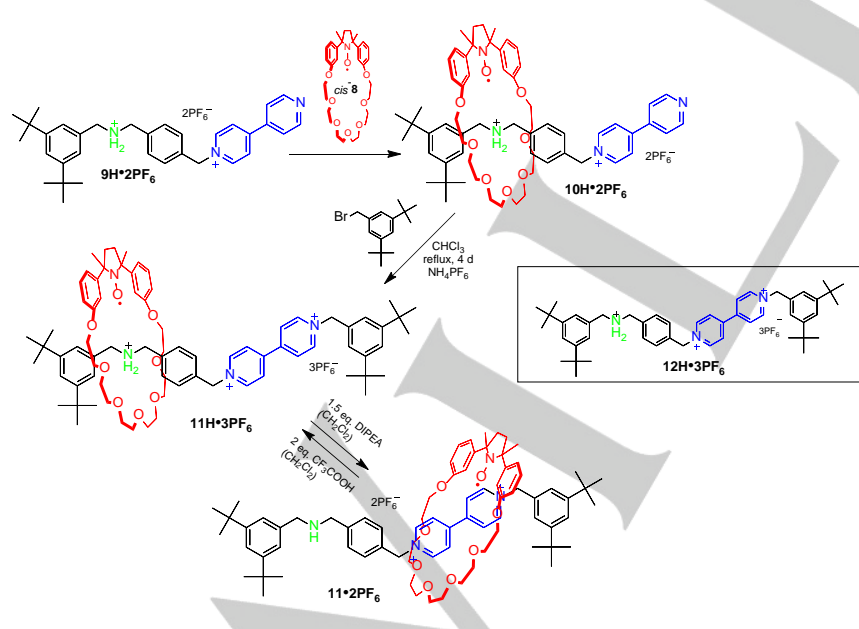

Scheme 3. Reaction scheme for the preparation of the rotaxane $11 \mathrm{H} \bullet 3 \mathrm{PF}_{6}$ and the switching process between $11 \mathrm{H} \cdot 3 \mathrm{PF}_{6}$ and $\mathbf{1 1} \cdot 2 \mathrm{PF}_{6}$

Evidence for the formation of pseudorotaxane in $\mathrm{CHCl}_{3}$ was obtained by observing complete solubilisation of the half- dumbbell $\mathbf{9 H} \cdot \mathbf{2} \mathrm{PF}_{6}$ which is almost completely insoluble in chlorinated hydrocarbons. The pseudorotaxane was then reacted with (3,5-di-tert-butyl)-benzyl bromide by $\mathrm{SN}_{2}$-mediated stoppering reaction to afford rotaxane $\mathbf{1 1 H}_{\mathbf{H}} \cdot 3 \mathrm{PF}_{6}$ in $30 \%$ yield. The rotaxane formation was detected by ESI-MS (see Supporting Information), UV-visible and EPR spectroscopy.

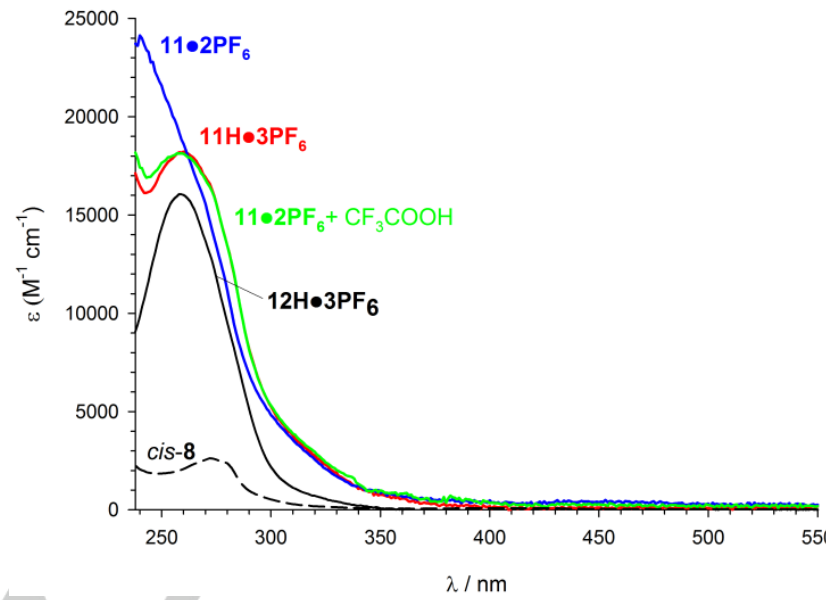

Figure 3. Absorption spectra of macrocycle cis-8 (dashed black line), free dumbbell $\mathbf{1 2} \cdot 2 \mathbf{P F}_{6}$ (black line) and rotaxanes $\mathbf{1 1} \mathbf{H} \cdot 3 \mathbf{P F}_{6}$ before (red line) and after subsequent addition of 1.5 eq of base (blue line) and $\mathrm{CF}_{3} \mathrm{COOH}$ (green line) in ACN at room $\mathrm{T}$

Figure 3 compares the absorption spectra of $11 \mathrm{H} \cdot 3 \mathrm{PF}_{6}$ and its constituents, i.e., the free dumbbell $12 \mathrm{H} \cdot 3 \mathrm{PF}_{6}$ and the macrocycle cis-8. The most intense absorption feature in the rotaxane arises from the viologen unit $\left(\varepsilon=18230 \mathrm{M}^{-1} \mathrm{~s}^{-1}, \lambda=259\right.$ $\mathrm{nm}$ ). This band overlaps with the weaker band due to the aromatic rings of the macrocycle unit and it is essentially unperturbed on going from $12 \mathrm{H} \bullet 3 \mathbf{P F}_{6}$ to $11 \mathrm{H} \bullet 3 \mathbf{P F}_{6}$. This spectral feature suggests that the macrocyle is not significantly interacting with the $\mathrm{BPY}^{2+}$ unit, consistent with it being localised predominantly over the ammonium site of the thread as expected.

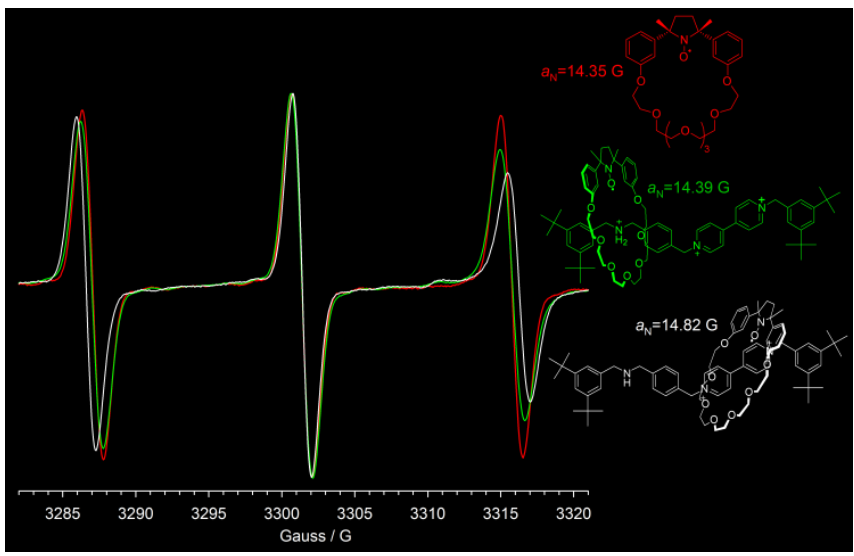

Figure 4. EPR spectra of macrocycle cis-8 (red line) and rotaxanes $\mathbf{1 1} \mathbf{H} \bullet \mathbf{3 P F}$ (green line) and $\mathbf{1 1 . 2} \mathbf{P F}_{6}$ (white line) in $\mathrm{CH}_{2} \mathrm{Cl}_{2}$ at room $\mathrm{T}$. 
Figure 4 (green line) shows the EPR spectrum of rotaxane 11H•3PF .recorded in $\mathrm{CH}_{2} \mathrm{Cl}_{2}$ at $298 \mathrm{~K}$, exhibiting typical nitroxide EPR signals with the high field line broadened due to restricted tumbling and, thus, incomplete averaging of the anisotropic components of the hyperfine and $g$-tensor. The $a_{N}$ hyperfine splitting and the rotational correlation times $\tau$, are reported in Table 1. ${ }^{[20]}$ The value of $a_{N}=14.39 \mathrm{G}$ is very close to that observed both for the free macrocycle and the dibenzylammonium@cis-8 complex. According to UV analysis, theses EPR data strengthens the conclusion that the macrocycle predominantly encircles the ammonium station. The higher affinity shown by cis-8 for the complexation of dibenzyl ammonium site compared to the $\mathrm{BPY}^{2+}$ one, is in line with the complexation behaviour shown by the related macrocycle dibenzo[24]crown-8 dibenzyl for which the association constant with dibenzyl ammonium cation is about 5 times higher $\left(K_{\text {eq }}=\right.$ $\left.420 \mathrm{M}^{-1}\right)^{[21]}$ than that measured with dibenzyl viologen $\left(K_{\text {eq }}=82\right.$ $\left.\mathrm{M}^{-1}\right)^{[19]}$ in $\mathrm{ACN}$ at $298 \mathrm{~K}$.

The rotational correlation time measured in the EPR spectrum of $1 \mathbf{1} \mathbf{H}_{\mathbf{3}} \mathbf{P F}_{\mathbf{6}}$ is 4.3 times higher than that measured in the spectrum of cis-8 (Table 1). This substantial reduction of the tumbling rate is typical of spin labels attached to larger molecules and can be considered a further indication of the formation of the MIM structure.

Table 1. EPR parameters of nitroxides in $\mathrm{CH}_{2} \mathrm{Cl}_{2}$ at 298K. [20]

\begin{tabular}{lccll}
\hline Nitroxide & $a_{N} / G$ & $\begin{array}{c}\text { Line width of } \\
\text { central line/ } G\end{array}$ & $\begin{array}{l}\text { Signal } \\
\text { ratio } h_{0} / h_{-1}\end{array}$ & $\begin{array}{l}\text { Correlation } \\
\text { time } \tau / s\end{array}$ \\
\hline cis-8 & 14.35 & 1.38 & 1.10 & $4.6 \times 10^{-11}$ \\
$\mathbf{1 1 H \bullet 3 P F} 6$ & 14.39 & 1.42 & 1.47 & $2.0 \times 10^{-10}$ \\
$\mathbf{1 1 . 2 \mathbf { P F } _ { 6 }}$ & 14.82 & 1.41 & 1.65 & $2.6 \times 10^{-10}$ \\
\hline
\end{tabular}

Although, we found some differences comparing the ${ }^{1} \mathrm{H}$ NMR data of the dumbbell and the rotaxane, ${ }^{[22]}$ NMR studies are not particularly useful for the characterization of $11 \mathrm{H} \bullet 3 \mathrm{PF}_{6}$ because most of the signals were broadened by the presence of the paramagnetic center. It is worth noting that attempts to reduce the rotaxane with phenylhydrazine to the diamagnetic hydroxylamine derivative were unsuccessful, denoting a considerable inertness of the nitroxidic interlocked assembly to this reaction conditions.

Thus, the shuttling of the macrocycle towards the secondary $\mathrm{BPY}^{2+}$ station (Scheme 3) was followed by UV-Visible and EPR spectroscopies. Treatment of rotaxane $11 \mathbf{H} \bullet 3 \mathrm{PF}_{\mathbf{6}}$ in $\mathrm{CH}_{2} \mathrm{Cl}_{2}$ with the non-nucleophilic diisopropyl-ethylamine ( $\mathrm{Pr}_{2} \mathrm{EtN}$ DIPEA), which is strong enough to deprotonate the $\mathrm{NH}_{2}{ }^{+}$ center, ${ }^{[23]}$ led to perturbation of the dominant viologen UVabsorption features around $259 \mathrm{~nm}$. As shown in Figure 3 (blue line), deprotonation is accompanied by a small blue shift and an increase in the absorbance of viologen band. This behavior is consistent with the displacement of cis-8 to predominantly encircle the bipyridinium station leading to a stabilization of the ground state of $\mathrm{BPY}^{2+}$ due to charge-dipolar interactions between this unit and the nitroxidic moiety In contrast, deprotonation of free dumbbell $12 \mathrm{H} \cdot 3 \mathrm{PF}_{6}$ did not cause appreciable variation in the corresponding absorption band (see Supporting Information). ${ }^{[24]}$

Finally, the addition of a stoichiometric amount of trifluoroacetic acid (TFA) to the rotaxane after the addition of the base caused the quantitative recovery of the initial absorption spectrum (see Figure 3, green line), consistent with the system returning to its state in which macrocycle predominantly encircles the ammonium station.

Addition of increasing amount of $i-\operatorname{Pr}_{2} \mathrm{EtN}$ to the rotaxane also produces a noticeable increase in the EPR nitrogen coupling which reaches a plateau after addition of 1 eq. of base $\left(a_{N}=14.82 G, \Delta a_{N}=+0.43 G\right.$, see Figure 4 , white line $)$. The increase in the value of $a_{N}$ is similar to that observed when free DBV is complexed by the macrocycle cis-8 (see Figure 2).

During the titration with $i-\mathrm{Pr}_{2} \mathrm{EtN}$, the protonated

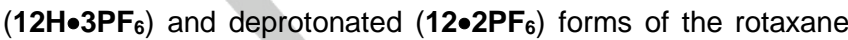
are present in solution simultaneously and in equilibrium with one another. It has been reported that, in a related diamagnetic rotaxane, base induced shuttling of a dibenzo[24]crown ether macrocycle to $\mathrm{BPY}^{2+}$ station is complete in seconds and acidinduced reverse of this process occurs on a timescale of tens of milliseconds. ${ }^{[25]}$ If we assume proton transfer between ${ }^{12} \mathbf{H} \bullet 3 P F_{6}$ and $12 \cdot 2 \mathrm{PF}_{6}$ takes place on similar time scales the exchange between the protonated and deprotonated states will be significantly slower than the EPR timescale. Thus, during the titration, the EPR spectrum is expected to be the superposition

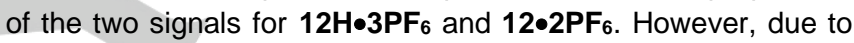
the large spectral line width, the detection of separate EPR signals is prevented and the variation in the relative amount of the radical co-conformations is expected to give rise only to a variation in the corresponding apparent field separation of the spectral lines.

Indeed, Figure 5 shows the changes in the field separation, $\Delta G$, between the central and high field lines, observed experimentally by EPR, upon addition of increasing amounts of $i-\mathrm{Pr}_{2} \mathrm{EtN}$ to $11 \mathrm{H} \bullet 3 \mathrm{PF}_{6}$ When $i-\mathrm{Pr}_{2} \mathrm{EtN}$ is less than 1 eq. (referred to the rotaxane) both nitroxides $11 \mathrm{H} \cdot 3 \mathrm{PF}_{6}$ and $\mathbf{1 1 \cdot 2 \mathrm { PF } _ { 6 }}$ are present and $\Delta G$ reflects the relative amount of protonated and deprotonated species.

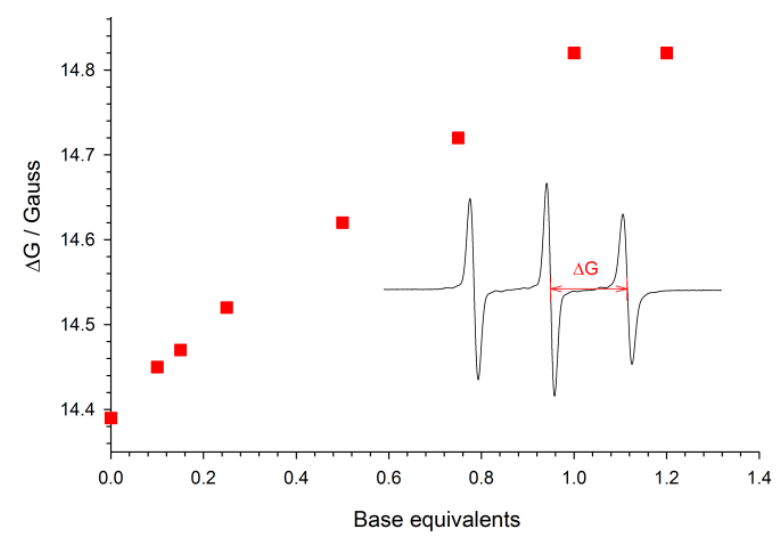

Figure 5. Field separation, $\Delta G$, between the central and high field lines, monitored by EPR, upon addition of $i-\mathrm{Pr}_{2} \mathrm{EtN}$ to $11 \mathrm{H} \bullet 3 \mathrm{PF}_{6}$. 
The switching of the rotaxane also leads to a slight increase of rotational correlation time $\tau$ from $2.0 \times 10^{-10}$ to $2.6 \times 10^{-10} \mathrm{~s}$ (see Figure 4 and Table 1 ). The change in the rotational correlation time when passing from one coconformation to the other can be associated to the reduced ability of the 'wheel' to rotate relative to the thread when is located at the $\mathrm{BPY}^{2+}$ station.

Conversely, the base addition performed on a 1:1 mixture of the non-interlocked rotaxane components (cis-8 and the free dumbbell, $\mathbf{1 2} \mathrm{H} \cdot 3 \mathrm{PF}_{6}$ ) did not cause variations in the nitrogen splitting nor in the correlation time. Such a control experiment confirms that the observed EPR spectral parameters changes arise from the interaction between the radical unit and the bipyridinium unit which become possible as a result of ring shuttling in the deprotonated rotaxane.

The addition of a stoichiometric amount of TFA after the addition of $i-\operatorname{Pr}_{2} E t N$ caused the quantitative recovery of the initial EPR spectrum ( $a_{N}=14.39 \mathrm{G}$, see Supporting Information) as a consequence of the macrocycle returning to predominantly encircle the ammonium station. The complete base- and acidinduced switching cycle of the EPR pattern was repeated several times without an appreciable loss of signal, highlighting the reversibility of the process.

\section{Conclusion}

In conclusion, we have reported the synthesis of the first nitroxide rotaxane in which the nitroxide unit acts not only as EPR probe for the switching, but interacts with a recognition site (bipyridinium) in the thread. While in previous work, the shuttling process was detected by EPR because of switching between non-coupled (three-line EPR spectrum) and coupled (five-line EPR spectrum) states of nitroxide radicals connected to the ring and dumbbell, in this work we have shown that a simple change in the nitrogen coupling reports on switching process between dialkylammonium and 4,4'-bipyridinium recognition sites. The nitroxide behaves as spin probe of shuttling process because is able to interact with bipyridinium unit by charge-dipolar interaction. Finally, the presence of nitroxide units provides an unusual functional addition to the diversity of templates available for assembling rotaxanes and could potentially lead to new generations of radical molecular machines and superstable radicals.

\section{Acknowledgements}

This work was supported by Alma Mater Studiorum - University of Bologna. E. K. thanks Erasmus program for financial support.

Keywords: rotaxane $\cdot$ nitroxide $\cdot$ electron paramagnetic resonance $\bullet$ spin probes $\bullet$ supramolecular chemistry

[1] M. M. Haugland, E. A. Anderson, J. E. Lovett, Electron Paramag. Reson., 2017, 25, 1-34.

[2] M. J. Davis, Methods, 2016, 109, 21-30 and references therein.

[3] For recent example, see H. Yu, L. Cao, F. Li, Q. Wu, Q. Li, S. Wang, Y. Guo, RSCAdv. 2015, 5, 63655-63661.
[4] For selected examples of the application, see a) M. Shibuya, S Nagasawa, Y. Osada, Y. Iwabuchi, J. Org. Chem. 2014, 79, 1025610268; b) J. Willwacher, A. Fürstner, Angew. Chem., Int. Ed. 2014, 53, 4217-4221; c) L. Hoffmeister, P. Persich, A. Fürstner, Chem. Eur. J. 2014, 20, 4396-4402.

[5] Nitroxide Mediated Polymerization: From Fundamentals to Applications in Materials Science (Ed.: D. Gigmes), The Royal Society of Chemistry, Cambridge, 2015.

[6] Magnetism: A Supramolecular Function (Ed.: O. Kahn), Kluwer Academic: Dordrecht, 1996.

[7] Comprehensive Supramolecular Chemistry (Ed:. J. M. Lehn), Pergamon, Oxford, 1996.

[8] N. Kocherginsky, H. M. Swartz in Nitroxide Spin Labels: Reactions in Biology and Chemistry (Eds.: N. Kocherginsky, H. M. Swartz), CRC press, Boca Raton, 1995, p 27.

[9] P. Franchi, M. Lucarini, P. Pedrielli, G. F. Pedulli, ChemPhysChem 2002, 3, 789-793.

[10] a) V. Mugnaini, C. Punta, R. Liantonio, P. Metrangolo, F. Recupero, G. Resnati, G. F. Pedulli, M. Lucarini, Tetrahedron Lett. 2006, 47, 32653269; b) G. R. Hanson, P. Jensen, J. McMurtrie, L. Rintoul, A. S Micallef, Chem. Eur. J. 2009, 15, 4156-4164; c) L. Gualandi, E. Mezzina, P. Franchi, M. Lucarini, Chem. Eur. J. 2016, 22, 16017-16021.

[11] J. L. Hodgson, M. Namazian, S. E. Bottle, M. L. Coote, J. Phys. Chem. A, 2007, 111, 13595-13605.

[12] a) G. Jeschke, A. Godt, ChemPhysChem 2003, 4, 1328-1334; b) P Franchi, M. Lucarini, G. F. Pedulli, Curr. Org. Chem. 2004, 8, 18311849; c) R. Pievo, C. Casati, P. Franchi, E. Mezzina, M. Bennati, M. Lucarini, ChemPhysChem 2012, 13, 2659-2661; d) C. Casati, P. Franchi, R. Pievo, E. Mezzina, M. Lucarini, J. Am. Chem. Soc. 2012 134, 19108-19117; e) R. Manoni, F. Romano, C. Casati, P. Franchi, E. Mezzina, M. Lucarini, Org. Chem. Front. 2014, 1, 477-483; f) V. Bleve, C. Schäfer, P. Franchi, S. Silvi, E. Mezzina, A. Credi, M. Lucarini, ChemistryOpen 2015, 4, 18-21 g) F. Romano, R. Manoni, P. Franchi, E. Mezzina, M. Lucarini, Chem. Eur. J. 2015, 21, 2775-2779; h) P. Franchi, V. Bleve, E. Mezzina, C. Schäfer, G. Ragazzon, M. Albertini, D. Carbonera, A. Credi, M. Di Valentin, M. Lucarini, Chem. Eur. J. 2016, 22, 8745-8750.

[13] As an example, see: a) A. Trabolsi, N. Khashab, A. C. Fahrenbach, D. C. Friedman, M. T. Colvin, K. K. Cotí, D. Benitez, E. Tkatchouk, J. C Olsen, M. E. Belowich, R. Carmielli, H. A. Khatib, W. A. Goddard III, M. R. Wasielewski, J. F. Stoddart, Nature Chem. 2010, 2, 42-49; b) J. C. Barnes, A. C. Fahrenbach, D. Cao, S. M. Dyar, M. Frasconi, M. A. Giesener, D. Benítez, E. Tkatchouk, O. Chernyashevskyy, W. H. Shin, H. Li, S. Sampath, C. L. Stern, A. A. Sarjeant, K. J. Hartlieb, Z. Liu, R. Carmieli, Y. Y. Botros, J. W. Choi, A. M. Z. Slawin, J. B. Ketterson, M. R. Wasielewski, W. A. Goddard III, J. F. Stoddart, Science 2013, 339, 429433.

[14] J. F. W. Keana, J. Cuomo, L. Lex, S. E. Seyedrezai, J. Org. Chem 1983, 48, 2647-2654.

[15] T. Shibata, K. Uemae, Y. Yamamoto, Tetrahedron: Asymmetry 2000, 11, 2339-2346.

[16] E. Mezzina, M. Fanì, F. Ferroni, P. Franchi, M. Menna, M. Lucarini, J. Org. Chem. 2006, 71, 3773-3777.

[17] T. D. Lee, G. B. Birrell, J. F. W. Keana, J. Am. Chem. Soc. 1978, 100 1618-1619.

[18] The rate of formation and breaking of the pseudorotaxane is expected to be very large on the time scale of EPR spectroscopy. Thus, the experimental nitrogen coupling $a_{N}$ represents the concentrationweighted average of the value due to the free and complexed species.

[19] A. B. Braunschweig, C. M. Ronconi, J.-Y. Han, F. Aricó, S. J. Cantrill, J. F. Stoddart, S. I. Khan, A. J. P. White, D. J. Williams, Eur. J. Org. Chem. 2006, 1857-1866.

[20] The rotational correlation time $\tau(\mathrm{s})$ was determined using the following equation: $\tau=6.5 \times 10^{-10} \times L W \times\left(\sqrt{h_{0} / h_{-1}}-1\right)$. Here $L W$ is the peakto-peak width (in Gauss) of the central line; $h_{0}$ and $h_{1}$ are the heights of the central and the highfield lines, respectively. 
[21] A. R. Williams, B. H. Northrop, K. N. Houk, J. F. Stoddart, D. J. Williams, Chem. Eur. J. 2004, 10, 5406-5421.

[22] Some difference observed in the H-NMR spectra were due to upfield shifts in the rotaxane of proton signals of bis-pyridinium moiety relative to those of the free rod (ca. 0.1-0.6 ppm) and $\left(\mathrm{CH}_{2}\right) \mathrm{NH}_{2}{ }^{+}$proton signals broadening in the rotaxane.

[23] M.-V. Martínez-Díaz, N. Spencer, J. F. Stoddart, Angew. Chem. Int. Ed. Engl. 1997, 36, 1904-1907.
[24] P. R. Ashton, R. Ballardini, V. Balzani, I. Baxter, A. Credi, M. C. T. Fyfe M. T. Gandolfi, M. Gûmez-Lûpez, M.-V. Mart.nez-D.az, A. Piersanti, N. Spencer, J. F. Stoddart, M. Venturi, A. J. P. White, D. J. Williams, J. Am. Chem. Soc. 1998, 120, 11932-11942.

[25] S. Garaudée, S. Silvi, M. Venturi, A. Credi, A. H. Flood, J. F. Stoddart, ChemPhysChem 2005, 6, 2145- 2152 


\section{Entry for the Table of Contents}

\section{COMMUNICATION}

The synthesis of a nitroxide crown ether and its use as the wheel in a bistable [2]rotaxane is reported. It is shown that the EPR read-out discriminates between dialkylammonium and 4,4'-bipyridinium recognition sites and this favourable feature can be used to probe shuttling process of the paramagnetic wheel in a bistable [2]rotaxane.

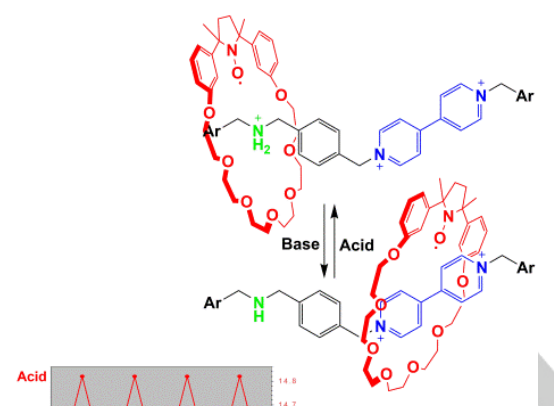

V. Bleve, P. Franchi, E. Konstanteli, L. Gualandi, S. M. Goldup, E. Mezzina, * M. Lucarini*

Page No. - Page No.

Synthesis and Characterization of a paramagnetic [2]Rotaxane based on a crown ether-like wheel incorporating a nitroxide motif 